\title{
The Technologies of Close-range Photogrammetry and Application in Manufacture
}

\author{
Xishuang Jing ${ }^{1, a}$, Chengyang Zhang ${ }^{1, b}$, Zhanlei Sun ${ }^{1, c}$, \\ Gang Zhao ${ }^{1, d}$, Yida Wang ${ }^{2, e}$
}

${ }^{1}$ School of Mechanical Engineering and Automation, Beihang University, Beijing, 100191, China

${ }^{2}$ PLA Military Representative Office in Shenyang Aircraft Corporation, Shenyang, 110034, China

atom@buaa.edu.cn, b'zhangchengyang@buaa.edu.cn, csunzhanlei@buaa.edu.cn,

dzhaog@163.com, eguohj_sy@163.com

Keywords: Close-range Photogrammetry Measuring Systems, Measurement Precision, Systematical Errors, Industrial Measurement.

Abstract. In this paper, the working principle and composition of close-range photogrammetry measuring systems are introduced and the general key technique in picking up measurement points and calibrating the camera are analyzed. The close-range photogrammetry measuring systems are compared with other major performance of the measuring systems. Besides, the applications in manufacturing and the shortcomings of close-range photogrammetry measuring systems are described in this paper.

\section{Introduction}

With the development of digital industry, the demand for the quality of industrial products is increasing, which puts more requires on the measuring accuracy and efficiency in its manufacturing process. As digital measuring systems, close-range photogrammetry measuring systems are able to detect object within dozens of meters as well as measuring tens of thousands of points at the same time, which can also guarantee the accuracy of the case. Such prosperities like high precision and high efficiency make it possible for close-range photogrammetry measuring systems to be widely used in industrial measurement.

\section{The components of close-range photogrammetry}

Close-range measuring system consists of corrected lens of camera, marked points and computer. Working principle is shown in Fig.1. Marked points are already pasted on the object to be measured. The camera takes a number of pictures towards objects from different directions, in order to generate two-dimensional digital images. The location of marked points can be identified in the computer by using image recognition technology. The coordinates of marked points, and position and orientation information of the camera can be obtained by using collinearity equation and mathematical model ${ }^{[1]}$.

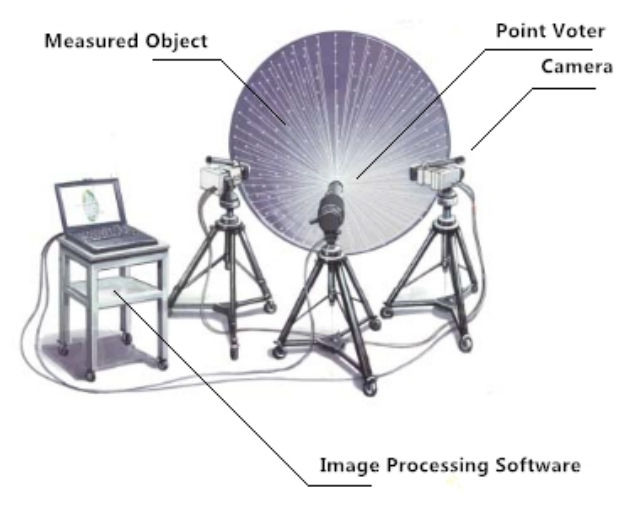

Fig. 1 Working principle of Close-range measuring system 
At present, there are many companies in the world launch their own close-range photogrammetry measuring systems. For example, GSI rolled out V-STARS photography three-coordinate measuring system based on high resolution and high precision solid state image sensors; In German, GOM company launched the TRITOP camera coordinate measuring systems Germany AICON 3D SYSTEMS GMBH launched DPA-PRO single-camera photogrammetric systems; Norway METRONOR launched METRONOR photoelectric type coordinate measuring system; Belgium METRIS Announced METRIS K600 on-line Photogrammetry System ${ }^{[1,2]}$. The comparison among the measuring systems above on measuring accuracy, range and characteristic are shown in Table 1.

Table1 Comparison among the existing measuring systems

\begin{tabular}{|c|c|c|l|}
\hline Name & accuracy & range & \multicolumn{1}{|c|}{ characteristic } \\
\hline V-STARS & $5 \mu \mathrm{m} / \mathrm{m}$ & $50 \mathrm{~m}$ & $\begin{array}{l}\text { High accuracy, wide measurement range, } \\
\text { wide application }\end{array}$ \\
\hline TRITOP & $10 \mu \mathrm{m} / \mathrm{m}$ & $10 \mathrm{~m}$ & Use an ordinary SLR camera sensor \\
\hline DPA-PRO & $15 \mu \mathrm{m} / \mathrm{m}$ & $10 \mathrm{~m}$ & $\begin{array}{l}\text { Measurement results can be imported to } \\
\text { PolyWorks, Metrolog, RapidForm and } \\
\text { Metrosoft for subsequent analysis }\end{array}$ \\
\hline METRONOR & $10 \mu \mathrm{m} / \mathrm{m}$ & $30 \mathrm{~m}$ & Can measure the hidden point \\
\hline METRIS K600 & $12 \mu \mathrm{m} / \mathrm{m}$ & $6 \mathrm{~m}$ & Can be tracked 100 points in real time \\
\hline
\end{tabular}

\section{The characteristics of close-range photogrammetry}

High measuring accuracy. In the single-camera measuring system, relative accuracy can reach up to $5 \mu \mathrm{m}+5 \mu \mathrm{m} / \mathrm{m}$ in the case of a high configuration.

High efficiency. Getting information on tens of thousands of data points to be tested within a short time.

Stable performance. In the case of measurements in an adverse environment such as vibration, high temperature, high pressure and hazardous environments, can still achieve high precision measurement. Measure without contact. Non-contact measurement can be achieved using PRO-SPORT vote point by point, the object is not damaged at all.

Compared with other industrial measuring systems, close-range photogrammetry measuring system has a unique advantage on measuring accuracy, range and efficiency. Comparison among main industrial measuring systems on measure performance is shown in Table 2.

Table 2 Comparison among main industrial measuring systems

\begin{tabular}{|c|c|c|}
\hline Name & Strength & Weakness \\
\hline $\begin{array}{c}\text { Close-range } \\
\text { photogrammetry } \\
\text { measuring system }\end{array}$ & $\begin{array}{c}\text { High precision, high } \\
\text { efficiency, good stability, } \\
\text { non-contact } \\
\text { measurement, wide } \\
\text { measurement range }\end{array}$ & Low degree of automation \\
\hline $\begin{array}{c}\text { Laser tracking measuring } \\
\text { system }\end{array}$ & High precision & $\begin{array}{c}\text { Low efficiency of } \\
\text { point-by-point measurement }\end{array}$ \\
\hline $\begin{array}{c}\text { Articulated coordinate } \\
\text { measuring system }\end{array}$ & $\begin{array}{c}\text { Measure hidden points } \\
\text { Low efficiency of }\end{array}$ \\
\hline iGPS & $\begin{array}{c}\text { wide measurement range, } \\
\text { high efficiency }\end{array}$ & Loint-by-point measurement \\
\hline
\end{tabular}




\section{The key technologies of close-range photogrammetry}

Camera Calibration Techniques. The mathematical methods are used in camera calibration technique to reduce camera lens distortion effect on the precision of the measurement results, which reduces errors of coordinates information when a three-dimension photo transforming into a two-dimension one. Camera lens distortion is mainly in the manufacturing process, due to reasons such as processing and assembly, resulting in the same axis of the lens can not reach full, so that light is refracted through the lens, makes the measurement result does not fully meet the collinear, that is the measured point, the lens center and the image point are not collinear ${ }^{[4,5]}$. For the distortion of camera, the mathematical models are used to compensate for its correction. Currently scholars have put forward various compensation proof of the mathematical model of the camera, such as 10-parameter model, polynomial models, finite element modeling, artificial neural networks ${ }^{[2,6-8]}$. These models, mainly depending on the camera lens distortion and distortion of regular intensity to different mathematical models in order to improve the accuracy of calibration. When using the camera for close-up measurement after correction for space rendezvous according to collinearity equation and algorithm, it can calculate the spatial coordinates of the point to be measured and the relative position and orientation of the camera information.

Measuring Points Recognition Technology.After filming of the object to be measured affixed with points, and these points can be read correctly taken from the digital images to markers which is a prerequisite for computing space coordinates of the object to be measured. Measuring point recognition technology mainly decrease image recognition signs point errors. Shoot out in a close-range measuring system of digital images are black and white, image processing software analyzes each pixel in the image grayscale values to identify landmarks. Because the point is made of reflective material, in the digital luminance value is larger than the surrounding objects, which makes it possible to recognize the pointer location. Scholars have put forward a lot of marking recognition algorithms, such as edge detection of Canny operator, directional scanning, recursive filling survey, morphology $y^{[9-11]}$. After picking up the location information of points, it will also calculate center coordinates of points. Currently there are also many calculation algorithm on center of points, like oval intends legitimate, gray weighted centroid method and die version match method ${ }^{[9-13]}$, these algorithm calculate the center points coordinates mainly through judging border shape and gray value of points. Oval intends legitimate is better for processing image containing noise points, gray weighted centroid method is good at processing points of image in a smaller radius.

Automatically Image Matching Technology.Close-range measurement system needs to measure objects from more than one location to shoot several images, image processing software match the same point automatically together in different images in order to calculate the space coordinates. To implement automatic image matching, it is a must to paste code around the object to be detected or measured. Coding is designed to be recognized by the image processing software with numbering pattern, point which has the same number of code will be automatically matched together in all digital images, so it will be able to find the same point in different position of the digital image. In close-range photogrammetry measurement technology, using pixel geometry to complete automatic matching of image commonly used with the epipolar constraint and epipolar constraint based matching algorithm $[9,14]$.

\section{The error analysis of close-range photogrammetry}

Camera resolution, noise and other factors affect the precision of the measurement results. Level of details the camera can capture is called the resolution, which is measured in pixels. The more effective pixels the image contained, the greater the resolution will be ${ }^{[15]}$. In close-range photogrammetry measuring systems, relative accuracy of measurement is the ratio between precision of pixel coordinates and image resolution ${ }^{[16]}$. It is clear that under certain conditions of the image coordinates accuracy, the higher camera's resolution is, the higher relative accuracy will be. The rough parts of the output image when CCD camera (CMOS) sensor receive light as signal is called the noise reduction of 
the camera, which is usually generated by electronic jamming. Camera noise generating extra pixels in the image, it will display additional color in the image. When identifying the points to be measured, image processing software will get the center coordinates based on gray distribution for example, in the identification of elliptic boundary fitting, noise affects the gray distribution of boundary, thus reducing the precision of boundary identification, so leads to calculation errors on a center point coordinate. Therefore, in close-range photogrammetry measuring systems, choosing cameras with high s/n ratio is quite common in order to reduce the noise effect on the precision of the measurement results.

Also, reference meter in the system affects the precision of the measurement results. In close-range photogrammetry measuring system, it is common to place one or more reference meters around the object to be detected. Besides, the reference meters own required exact length and the precisions are also known, to ensure the coordinate system is very accurate in the results of measuring. Reference meter error, thermal stress and deformation will have an impact on measurement accuracy. Therefore, in order to reduce the error resulted from reference meter, using warp-proof materials in manufacturing works and using reference meter with high precision laser tracker in length measurement.

\section{Application in Manufacturing Industry}

Aerospace Industry.Aviation manufacturing parts have a high demand for precision of machining and assembly, besides, the geometry part is more complicated. According to close-range photogrammetry measurement, components which has been processed or assembled can be confirmed whether meet the quality requirements. The V-STARS measuring system is used to measure the Boeing 777 wing shown in Fig.2.

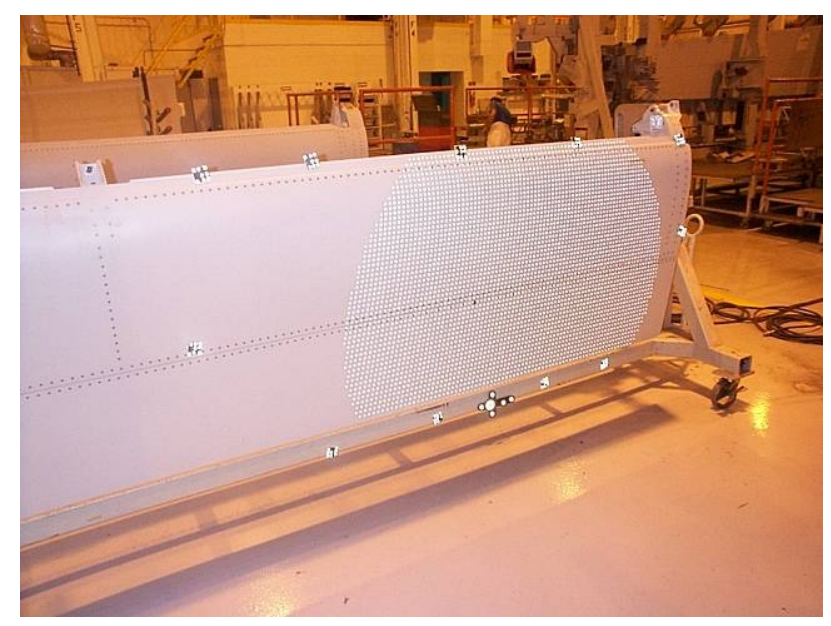

Fig.2 Measurement on the Boeing 777 wing

Aerospace Industry.Aerospace manufacturing products work and test in a low temperature vacuum environment because of its special working environment. General measuring equipment, such as laser trackers and iGPS, which need artificial target detection rods placed in the object ball. However, this process is difficult to achieve in a vacuum environment. With non-contact measurement of close-range measuring system, it is possible to measure object in a long distance successfully. European Space Agency used close-range measuring system measuring the deformation of astronomical telescope in low-temperature vacuum environment shown in Fig.3. 


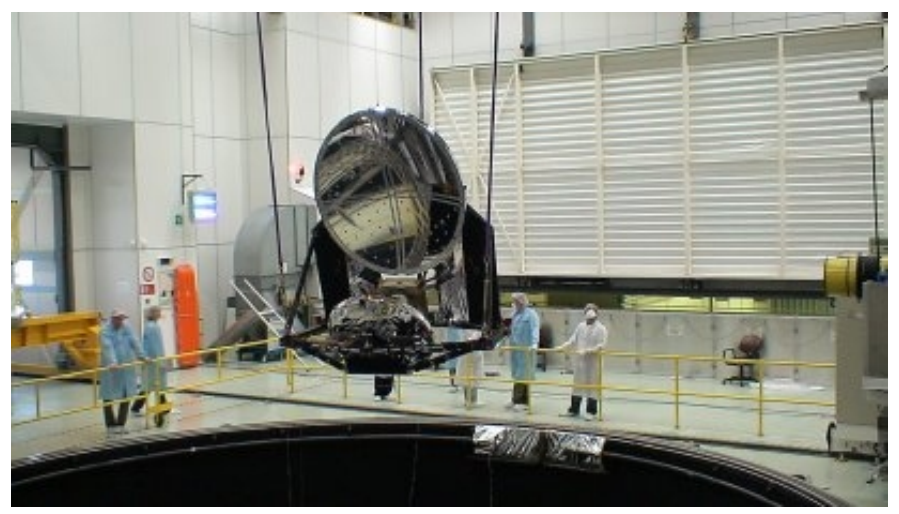

Fig.3 European Space Agency measure the deformation of astronomical telescope

Automotive Industry.In the automotive industry, product parts usually need for mass production and high production efficiency. It is available to complete the pointing and measurement of large auto parts by using close-range photogrammetry measuring system and online measuring system, thus improving vehicle productivity. Usage of close-range measuring system for BMW X5 BMW series automobile side panels is shown in Fig.4.

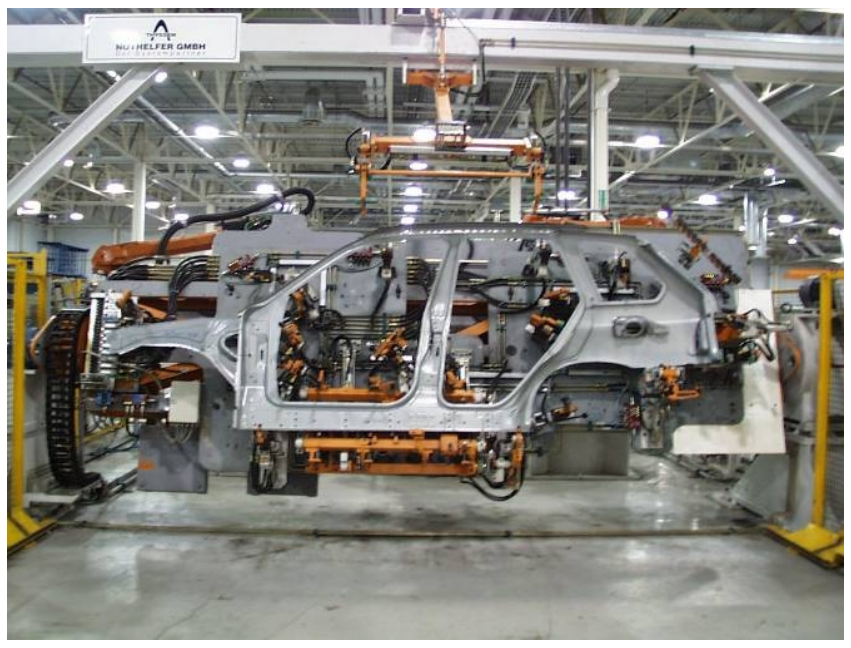

Fig.4 Measurement on BMW X5 BMW series automobile side panels

Ship Manufacturing.Compared with other industries, shipbuilding product is diverse by its large size. Close-range measuring system can guarantee the accuracy of the measurement range of dozens of meters, so it is an ideal tool for ship parts in the dimensional measurement. Japan IHI Corporation measure the shipping component radial line is shown in Fig.5

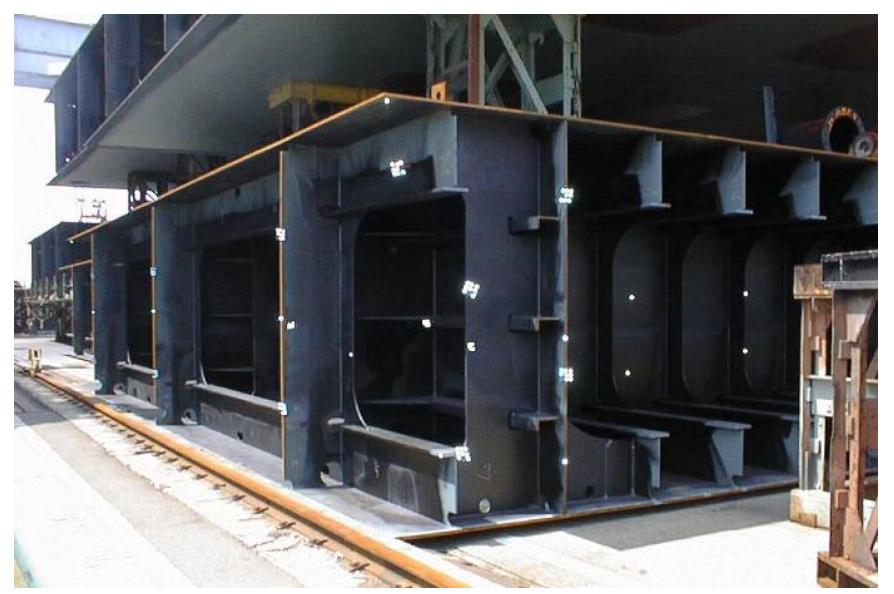

Fig.5 Japan IHI Corporation measure the shipping component radial line 


\section{Inadequacy of close-range photogrammetry}

Close-range photogrammetry measuring systems compared to other measuring systems, have the characteristics of high precision, high efficiency and high stability, it has been widely used in industrial measurement. But in the measure of manufacturing production, application of close-range photogrammetry measuring system limitations still exist.

Low Level of Automation. When close-range measuring system operates in a stand-alone offline measurements, it is required to paste code points at the object to be detected in advance, the bigger the object to be detected is, the more sticking points are, thus leading the more manual work which will reduce the efficiency of measurement greatly. Meanwhile, photo process is manual which causes a lower degree of automation.

Unable to Measure Small Objects. Close-range photogrammetry measuring systems usually require maintaining more than 1 meter of distance between the object to be detected and the camera because measurement results can be achieved to ensure best accuracy. It is unavailable for cramped space to be measured because it did not have enough measurements of space, and cannot be measured. So close-range photogrammetry measuring systems applicable to measurements on the larger space.

\section{Future Prospects}

Close-range photogrammetry measuring systems have been developed for several decades since its advent in the 30's of the last century. Close-range measuring system both innovated rapidly in hardware device and software algorithm ${ }^{[17]}$. In recent years, domestic universities, companies are constantly introducing advanced foreign technology, and gradually developed domestic close-range measurement technology ${ }^{[18-20]}$. Future development of close-range measuring system in industrial measurement can be summarized as the following.

Application in Docking Procedure of Large Parts. Characteristics of dynamic measurement based on close-range measuring system can monitor the process of docking such as aircraft, ships, automobiles and other components. By comparing the measuring unit current location coordinates and the final docking locations, it is available to compute components the direction and distance needed to move, so as to ensure the accuracy of docking.

Application in Automatically Drilling and Riveting Robots. It will be impossible for measuring system to output automatic drilling and riveting point coordinate in real time and guide the completion of drilling and riveting work. Close-range photogrammetry measuring systems measure the trajectory of robots need to move, and guides it automatically move to the specified location. Meanwhile, it can monitor the center point coordinates of robot machine tool, so as to ensure the positioning accuracy of drilling and riveting work station.

Application in Surveillance Running Process. Close-range photogrammetry measuring system will measure the process such as airplanes taking off and landing of flights and ships sailing, thus analyzing performance, deformation and other information of all parts of aircraft and ships during operation.

\section{References}

[1] G.P. Huang. Study on the key technologies of digital close range industrial photogrammetry and applications[D].Tianjin University, 2005.(In Chinese)

[2] Q.Q. Feng. Research and practice of digital industrial photogrammetry [D].PLA Information Engineering University, 2010. (In Chinese)

[3] Q. Yong. The basic technologies and application of close-range photogrammetry [J]. Intelligence, 2013, 19:190. (In Chinese) 
[4] S. Robson, M. R. Shortis. Practical influences of geometric and radiometric image quality provided by different digital camera systems [J]. The Photogrammetric Record, 2003, 16(92):225-248.

[5] F. Remondino, C Fraser. Digital camera calibration methods: considerations and comparisons [J]. International Archives of Photogrammetry, Remote Sensing and Spatial Information Sciences, 2006, 36(5): 266-272.

[6] W.H. Feng, H.L. Shang, W.G. Hou. Model of digital distortion image [J]. Geomatics and Information Science of Wuhan University, 2006, 02:99-103. (In Chinese)

[7] Kavzoglu T and Karsli F. Calibration of a digital sigle lens reflex(SLR) camera using artific neural networks[J]. IAPRS,2008, Vol.36(B5): 27-32.

[8] Tecklenburg W, Luhmann T, Hastedt H. Camera modelling with image-variant parameters and finite elements[C]. Heidelberg:2001:

[9] Otepka JO, Hanley HB, Fraser CS (2002) Algorithm developments for automated off-line vision metrology[J]. Int Arch Photogramm Remote Sens 5:60-67

[10]Clarke T A. An analysis of the properties of targets used in digital close range photogrammetric measurement[J]. Videometrics III,1994, Vol.SPIE Vol.2350: 251-262.

[11]Shortis M R, Clarke T A, and Short T. A comparison of some techniques for the subpixel location of discrete target images[J]. Videometrics III,1994, Vol.SPIE Vol.2350: 239-250.

[12]Otepka J. Precision target mensuration in vision metrology [D]. Technische Universitaat Wien,2004.

[13]Anchini R, Beraldin J -, and Liguori C. Subpixel location of discrete target images in close-range camera calibration: a novel approach[J]. Proc. SPIE, 2007, Vol.6491(Videometrics IX).

[14]Ariyawansa D D A P and Clarke T A. High speed correspondence for object recognition and tracking[J]. SPIE 1997, Vol.3174(Videometrics V): 70-79.

[15]W.H. Feng. Close-range photogrammetry [M]. Wuhan, China: Wuhan University Press, 2001. (In Chinese)

[16]Uffenkamp V. State of the art of high precision industrial photogrammetry [C]. In Third international workshop on accelerator alignment.Annecy, France:1993: 153-165.

[17]L. Wang. Theory research and practice of digital close range photogrammetry[D]. PLA

Information Engineering University, 2002. (In Chinese)

[18]Y.G. He, X.M. Wang, Z.Q. Zeng. Application of digital close range photogrammetry system[J].

Mining and Metallurgical Engineering, 2001, 04: 1-3. (In Chinese)

[19]M.F. Zhao. B.X. Shen, X.M. Wu, W.J. Wu, S.H. Wang. Application of rapid ambiguity-avoiding feature matching in multi-view stereo vision inspection system [J]. Journal of China Institute of Metrology, 2003, 02: 9-13. (In Chinese)

[20]S.J. Jia, J.T. Yu. Application of digital close-range photogrammetry to inspection of machine parts[J]. Acta Geodaetica et Cartographica Sinica, 2002, S1: 61-65. (In Chinese) 